\title{
Continuous blood pressure recordings simultaneously with functional brain imaging: studies of the glymphatic system
}

\begin{abstract}
Aleksandra Zienkiewicz, Niko Huotari, Lauri Raitamaa, Ville Raatikainen, Hany Ferdinando, et al.
\end{abstract}

Aleksandra Zienkiewicz, Niko Huotari, Lauri Raitamaa, Ville Raatikainen, Hany Ferdinando, Erkki Vihriälä, Vesa Korhonen, Teemu Myllylä, Vesa Kiviniemi M.D., "Continuous blood pressure recordings simultaneously with functional brain imaging: studies of the glymphatic system," Proc. SPIE 10063, Dynamics and Fluctuations in Biomedical Photonics XIV, 1006311 (3 March 2017); doi: 10.1117/12.2252032 


\title{
Continuous blood pressure recordings simultaneously with functional brain imaging - studies of the glymphatic system
}

\author{
Aleksandra Zienkiewicz*a ${ }^{* a}$ Niko Huotari ${ }^{\mathrm{b}}$, Lauri Raitamaa ${ }^{\mathrm{b}}$, Ville Raatikainen ${ }^{\mathrm{b}}$, \\ Hany Ferdinando ${ }^{\mathrm{a}}$, Erkki Vihriälä ${ }^{\mathrm{a}}$, Vesa Korhonen ${ }^{\mathrm{b}, \mathrm{c}}$, Teemu Myllyläa ${ }^{\mathrm{a}}$, Vesa Kiviniemi ${ }^{\mathrm{b}, \mathrm{c}}$ \\ ${ }^{a}$ University of Oulu, Optoelectronics and Measurement Techniques Unit, 3 Erkki Koiso- \\ Kanttilankatu, Oulu 90570, Finland; ${ }^{\mathrm{b}}$ University of Oulu, the Faculty of Medicine, Research Unit of \\ Medical Imaging, Physics and Technology, Oulu, Finland; ${ }^{\mathrm{c}}$ Oulu University Hospital, Medical \\ Research Center (MRC), Department of Diagnostic Radiology, Oulu, Finland, P.O. Box 50, Oulu \\ 90029 OYS, Finland;
}

\begin{abstract}
The lymph system is responsible for cleaning the tissues of metabolic waste products, soluble proteins and other harmful fluids etc. Lymph flow in the body is driven by body movements and muscle contractions. Moreover, it is indirectly dependent on the cardiovascular system, where the heart beat and blood pressure maintain force of pressure in lymphatic channels. Over the last few years, studies revealed that the brain contains the so-called glymphatic system, which is the counterpart of the systemic lymphatic system in the brain. Similarly, the flow in the glymphatic system is assumed to be mostly driven by physiological pulsations such as cardiovascular pulses. Thus, continuous measurement of blood pressure and heart function simultaneously with functional brain imaging is of great interest, particularly in studies of the glymphatic system.

We present our MRI compatible optics based sensing system for continuous blood pressure measurement and show our current results on the effects of blood pressure variations on cerebral brain dynamics, with a focus on the glymphatic system. Blood pressure was measured simultaneously with near-infrared spectroscopy (NIRS) combined with an ultrafast functional brain imaging (fMRI) sequence magnetic resonance encephalography (MREG, 3D brain $10 \mathrm{~Hz}$ sampling rate).
\end{abstract}

Keywords: glymphatic system, blood pressure, multimodal brain imaging, near-infrared spectroscopy, MRI

\section{INTRODUCTION}

The lymph system is an essential tool used to ensure tissue homeostasis. Lymph fluid consists of soluble materials and cells, collected from the interstitial space, which are cleaned in the lymph nodes and returned to the general circulation [1]. Lymphatic vessels are present throughout the body, except of the brain and spinal cord. Lack of conventional lymphatic system in central nervous system (CNS) was investigated by a team led by Maiken Nedergaard from the University of Rochester Medical Center. In a study published in 2012, they identified a functional waste clearance pathway, which uses the cerebrospinal fluid (CSF) to remove metabolic wastes and neurotoxins from the brain along paravascular channels [2][3]. This pathway was called a 'glymphatic system' (GS), where the word 'glymphatic' derives from the system's reliance on glial cells, and its functional resemblance to the lymphatic system [2]. Most of the glymphatic clearance activity occurs during sleep, which is an interesting insight on the restorative function of sleep as a time of enhanced removal of potentially neurotoxic waste products accumulated in CNS [4]. In recently published studies, Nedergaard's research team claims that besides waste elimination, the glymphatic system might also serve to distribute non-waste compounds such as lipids and glucose within the brain [5]. Very recently, it has been suggested that cardiac pulsations are not the only mechanism of glymphatic propulsion but also respiratory and very low frequency pulsations affect [6].

Correlation between GS impairment and various brain diseases (e.g. Alzheimer disease) is nowadays widely studied [5][7]. Since the CSF flux is driven by arterial pulsation, cardiovascular system (CVS) abnormalities (e.g. as increased vascular stiffness, decreased vascular tone) might affect brain waste clearance [3]. Thus, simultaneous measurement of GS and CVS is of great interest. In presented study, the setup consisting of continuous non-invasive blood pressure

Dynamics and Fluctuations in Biomedical Photonics XIV, edited by Valery V. Tuchin, Kirill V. Larin,

Martin J. Leahy, Ruikang K. Wang, Proc. of SPIE Vol. 10063, 1006311 · C 2017 SPIE

CCC code: $1605-7422 / 17 / \$ 18 \cdot$ doi: $10.1117 / 12.2252032$ 
monitoring (cNIBP) combined with NIRS and fMRI was tested in order to develop reliable method of these two systems simultaneous observation.

*aleksandra.zienkiewicz@oulu.fi

\section{METHOD OF MEASUREMENT AND ANALYSIS}

\section{1 cNIBP}

Continuous monitoring of blood pressure oscillations was performed using cNIBP device presented in Myllylä et al. [8]. The setup consists of two small opto-mechanical MRI compatible sensors connected with optical fibres to the electronics module placed outside MRI chamber [8]. During the measurement, one sensor is placed on patient's chest and the other on patient's neck, above the carotid artery. Each heartbeat results in tiny skin vibrations, caused by the blood pulsation. The movement is detected by the sensors and because of their spatial separation, there is a time delay between a response inducement in each sensor. This delay is called Pulse Transit Time (PTT) - time between two pulse waves propagating on the same cardiac cycle from two separate arterial sites [9]. PTT and corresponding Pulse Wave Velocity (PWV) were shown to be correlated with blood pressure [9][10]. Example of the pulsation detected by described sensors with marked PTT is shown on Figure 1.

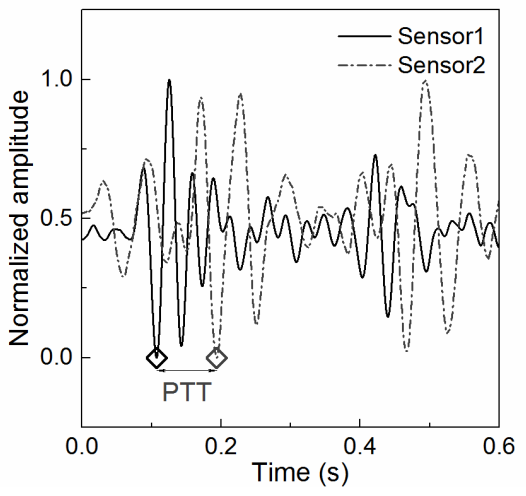

Figure 1 Blood pulsation detected by sensors in two separate placements. Both systolic and diastolic pulse can be distinguished. Markers indicate peaks used for PTT calculation.

The sampling rate of the cNIBP data was $10 \mathrm{kHz}$, to ensure high temporal accuracy. Conversion from PTT to BP was based on the method and formulae described by Gesche [9]. In this study only BP oscillations were of interest, not its absolute values. During the measurement sessions the patient had to perform breathing tasks in order to force a visible blood pressure change in the body. There were two kind of breathing tasks: breath holding (BH) together with deep and fast breathing (hyperventilation, HV), which were done several times for fixed duration. On Figure 2, there are shown corresponding breathing patterns for respCO $\mathrm{C}_{2}$, which were recorded simultaneously by an MRI compatible anesthesia monitoring device.

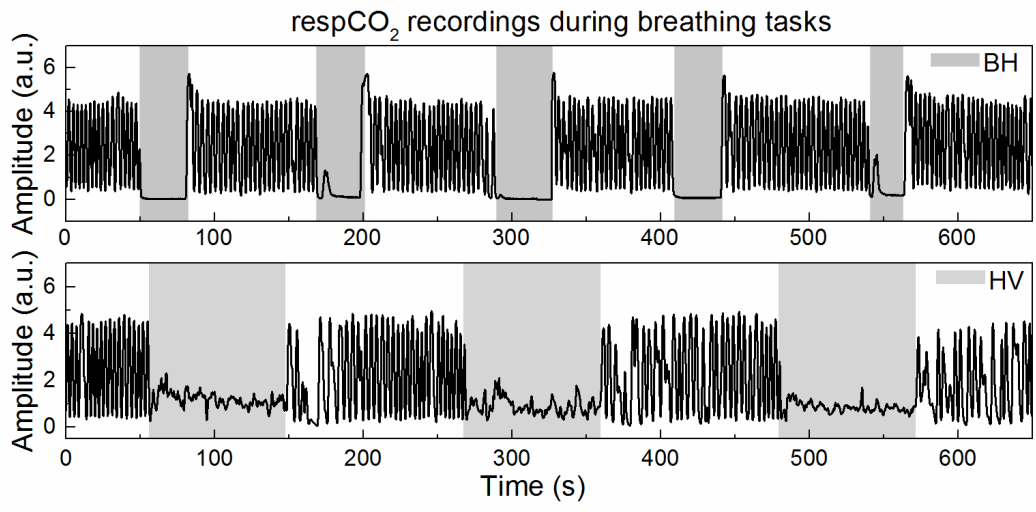

Figure $2 \mathrm{RespCO}_{2}$ breathing patterns recorded during $\mathrm{BH}$ task and $\mathrm{HV}$ task. 
Primary challenge faced during presented signals is accurate peak detection. Since the working principle of the sensors is based on proper acceleration measurement, they are especially sensitive to motion artifacts. In addition, neck is much softer place than chest and if the sensor is not attached tightly, it tends to reflect skin surface vibration. Figure 3 presents a comparison between signals recorded during normal breathing, breath hold and hyperventilation. Higher noise level in signals during HV might affect the peak detection accuracy and thus lower accuracy of estimated blood pressure oscillation.
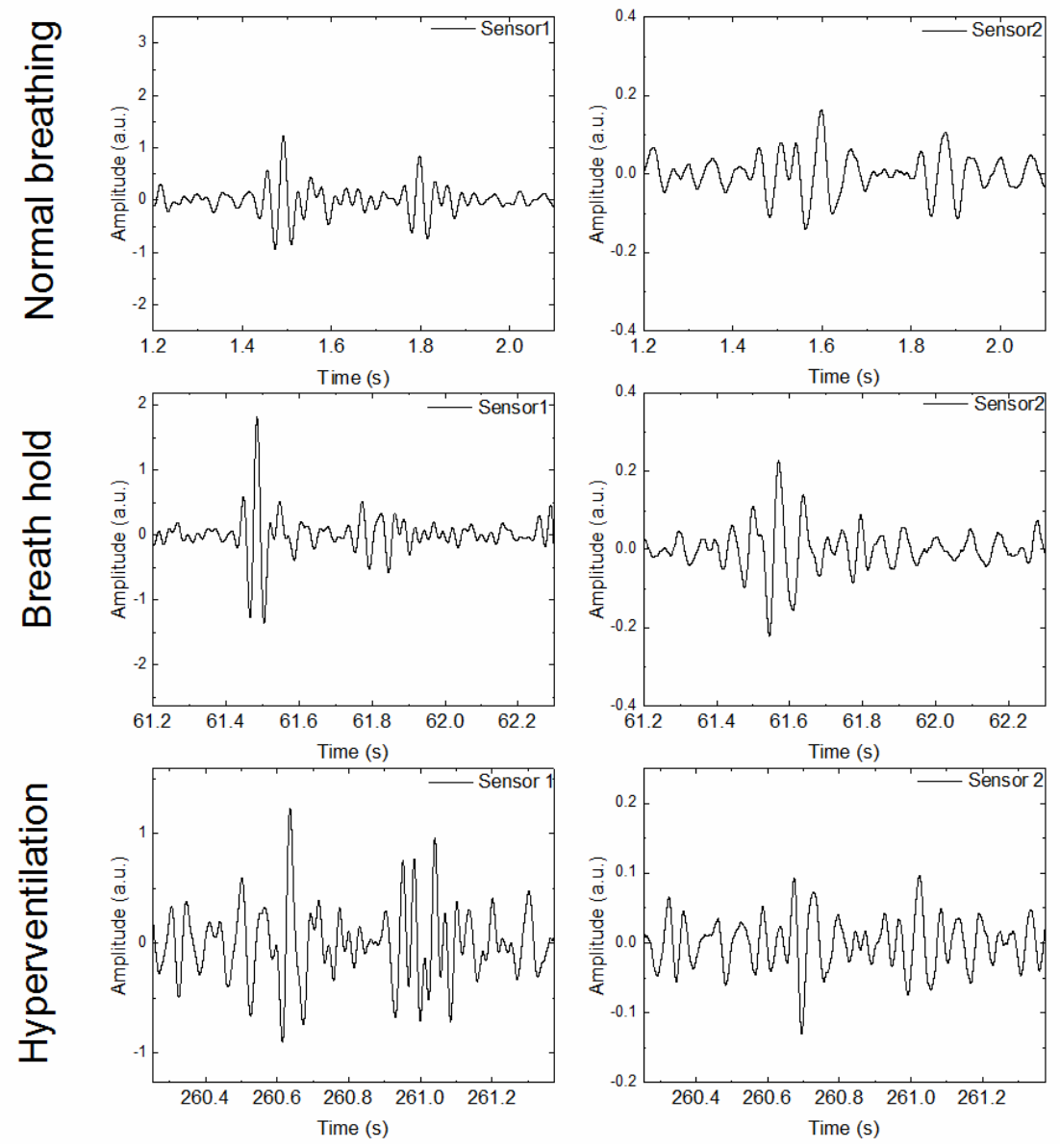

Figure 3. Signal shape differences as a result of breathing tasks.

\subsection{NIRS}

Cerebral hemodynamics were measured using NIRS technique presented in publications [11][12]. Similarly to cNIBP device, in order to ensure MRI compatibility, only optical fibres were brought inside MRI chamber while the NIRS device was outside MRI. Raw NIRS time series (wavelengths of $660 \mathrm{~nm}$ and $830 \mathrm{~nm}$ ) were measured from forehead (above left eye) and concentration change of total hemoglobin (HbT) was calculated using Matlab-based NIRS data analysis tool HoMer2, presented for instance in the publication by Huppert et al. [13]. The sampling rate of the NIRS data acquisition was $1 \mathrm{kHz}$.

\section{3 fMRI}

The subjects were scanned using Siemens 3T SKYRA MRI scanner with a 32-channel coil. We utilized an ultrafast fMRI sequence MREG, which was obtained from Freiburg University via collaboration with Jürgen Hennig's group [14][15]. MREG is a three dimensional spiral, single-shot sequence that under samples 3D k-space trajectory [16]. It allows approximately 20 times faster scanning than conventional fMRI, up to $10 \mathrm{~Hz}$. The following sequence parameters for MREG were used: repetition time $(\mathrm{TR})=100 \mathrm{~ms}$, echo time $(\mathrm{TE})=36 \mathrm{~ms}$, flip angle $(\mathrm{FA})=5^{\mathrm{o}}$. 
MREG data was preprocessed with typical FSL pipeline. T1-relaxation effects were minimized by removing one hundred time points from the beginning of each scan. Head motion correction was done utilizing FSL 5.08 MCFLIRT software [17]. After motion correction, the brain extraction was done by FSL 5.08 BET software. Three dimensional MPRAGE images were used to register MREG data in to 4-mm MNI152 standard space in prior to group independent component analysis (ICA). Finally, the resting state networks (RSNs) were separated by group probabilistic ICA as implemented in MELODIC [18]. Model order of 70 was chosen for the network separation.

\section{RESULTS}

On Figure 4 are presented continuous measurements of blood pressure, NIRS and MREG for one subject during BH task (left) and HV task (right). Pictures on the top represent z-statistic maps of the selected group ICA component registered to the 4-mm MNI space (sagittal, coronal and axial slices). The first signal plots show the time series of the selected group IC components which corresponds to the area shown in the top picture. Second plots show NIBP time series and the third ones are the NIRS signals (HbT). These group IC components (IC47 on both cases, coincidentally) were selected because they gave the highest positive correlation coefficient, $r$, to corresponding NIBP time series (for BH $r=$ 0.73 and for $\mathrm{HV} \mathrm{r}=0.48$ ). In total there were 70 different MREG components calculated.
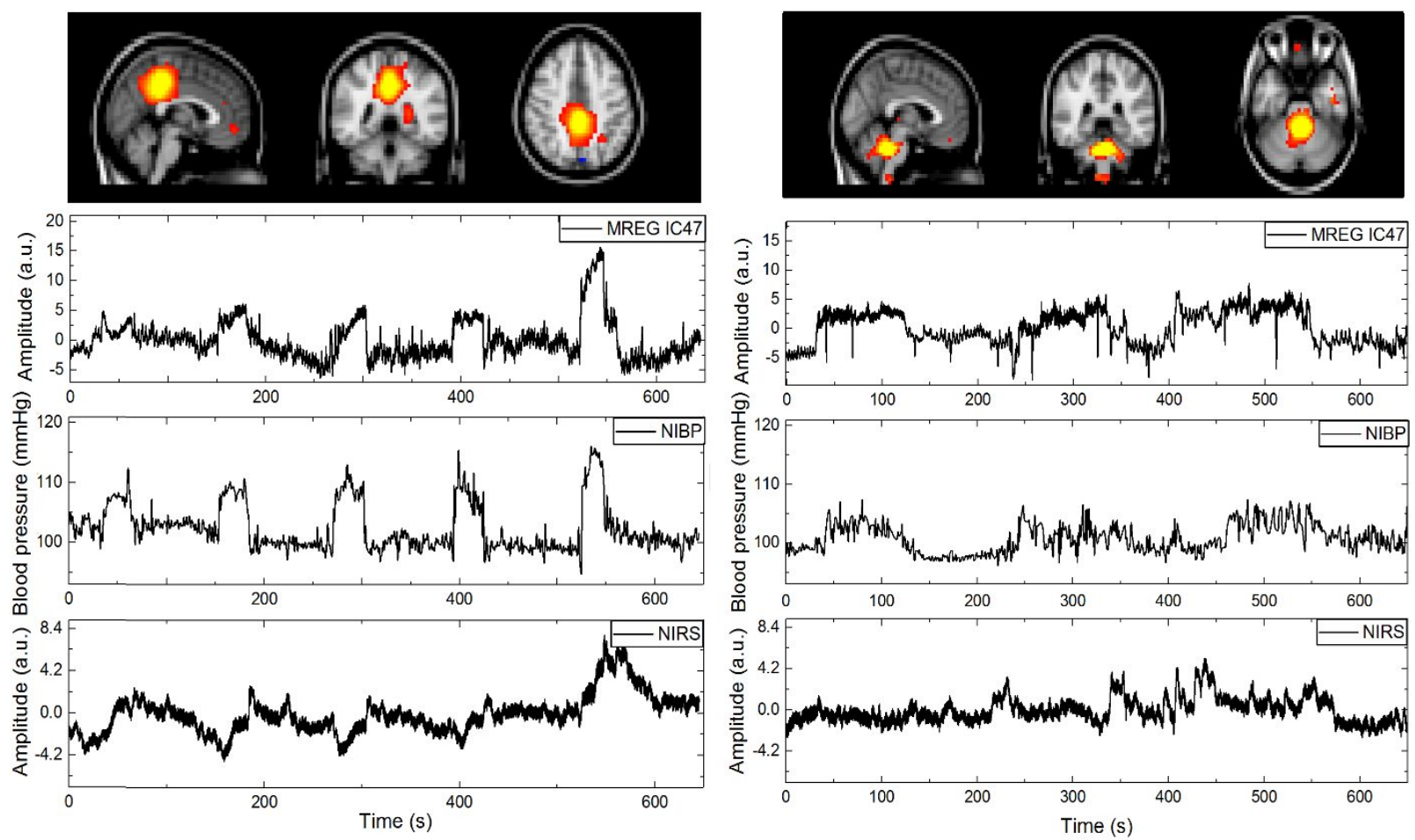

Figure 4. Example of signals recorded during BH task (left) and HV task (right). In the anatomical images on the top the MREG component 47 is shown, which has the highest correlation to blood pressure oscillations (NIBP) in both tasks.

For BH task Pearson's correlation coefficient $r=0.73$, for HV task: $r=0.48$.

\section{CONCLUSION}

Presented results show that our MRI compatible optics based sensing system for continuous blood pressure measurement can be used to detect correlating MREG components out of 70 different components. When using for instance different task-based experiments causing strong fluctuations in blood pressure it is possible to find MREG based brain areas that are most affected by these changes. Since the GS waste clearance mechanism derive most of it's power from cardiovascular pulsations, the detected high correlations between BP and MREG signal suggests that cNIBP might offer an external measures of glymphatic pulsations. 


\section{ACKNOWLEDGEMENTS}

This study was partially supported by Academy of Finland \& JAES-grants.

\section{REFERENCES}

[1] Liao, S. and Padera, T. P., "Lymphatic function and immune regulation in health and disease," Lymphatic research and biology 11(3), 136-143 (2013).

[2] Iliff, J. J., Wang, M., Liao, Y., Plogg, B. A., Peng, W., Gundersen, G. A., Benveniste, H., Vates, G. E., Deane, R., Goldman, S. A., Nagelhus, E. A. and Nedergaard, M. , "A paravascular pathway facilitates CSF flow through the brain parenchyma and the clearance of interstitial solutes, including amyloid beta," Sci.Transl.Med. 4(147), 147ra111 (2012).

[3] Venkat, P., Chopp, M. and Chen, J., "New insights into coupling and uncoupling of cerebral blood flow and metabolism in the brain," Croat.Med.J. 57(3), 223-228 (2016).

[4] Xie, L., Kang, H., Xu, Q., Chen, M. J., Liao, Y., Thiyagarajan, M., O'Donnell, J., Christensen, D. J., Nicholson, C., Iliff, J. J., Takano, T., Deane, R. and Nedergaard, M. , "Sleep drives metabolite clearance from the adult brain," Science 342(6156), 373-377 (2013).

[5] Jessen, N. A., Munk, A. S. F., Lundgaard, I. and Nedergaard, M. , "The glymphatic system: a beginner's guide," Neurochem.Res. 40(12), 2583-2599 (2015).

[6] Kiviniemi, V., Wang, X., Korhonen, V., Keinänen, T., Tuovinen, T., Autio, J., LeVan, P., Keilholz, S., Zang, Y-F., Hennig, J. and Nedergaard M., "Ultra-fast magnetic resonance encephalography of physiological brain activity Glymphatic pulsation mechanisms?," Journal of Cerebral Blood Flow \& Metabolism 36(6), 1033-45 (2016).

[7] Tarasoff-Conway, J. M., Carare, R. O., Osorio, R. S., Glodzik, L., Butler, T., Fieremans, E., Axel, L., Rusinek, H., Nicholson, C. and Zlokovic, B. V. , "Clearance systems in the brain [mdash] implications for Alzheimer disease," Nature Reviews Neurology 11(8), 457-470 (2015).

[8] Myllylä, T. S., Elseoud, A. A., Sorvoja, H. S., Myllylä, R. A., Harja, J. M., Nikkinen, J., Tervonen, O. and Kiviniemi, V. , "Fibre optic sensor for non-invasive monitoring of blood pressure during MRI scanning," Journal of biophotonics 4(1-2), 98-107 (2011).

[9] Gesche, H., Grosskurth, D., Küchler, G. and Patzak, A., "Continuous blood pressure measurement by using the pulse transit time: comparison to a cuff-based method," Eur.J.Appl.Physiol. 112(1), 309-315 (2012).

[10] Poon, C. and Zhang, Y., "Cuff-less and noninvasive measurements of arterial blood pressure by pulse transit time," 2005 IEEE Engineering in Medicine and Biology 27th Annual Conference, 5877-5880 (2006).

[11] Sorvoja, H., Myllylä, T., Kirillin, M. Y., Sergeeva, E. A., Myllylä, R. A., Elseoud, A., Nikkinen, J., Tervonen, O. and Kiviniemi, V., "Non-invasive, MRI-compatible fibreoptic device for functional near-IR reflectometry of human brain," Quantum electronics 40(12), 1067 (2010).

[12] Myllylä, T., Korhonen, V., Suraźyński, Ł., Zienkiewicz, A., Sorvoja, H. and Myllylä, R. , "Measurement of cerebral blood flow and metabolism using high power light-emitting diodes," Measurement 58, 387-393 (2014).

[13] Huppert, T. J., Diamond, S. G., Franceschini, M. A. and Boas, D. A. , "HomER: a review of time-series analysis methods for near-infrared spectroscopy of the brain," Appl.Opt. 48(10), D280-D298 (2009).

[14] Lee, H., Zahneisen, B., Hugger, T., LeVan, P. and Hennig, J. , "Tracking dynamic resting-state networks at higher frequencies using MR-encephalography," Neuroimage 65, 216-222 (2013).

[15] Zahneisen, B., Hugger, T., Lee, K. J., LeVan, P., Reisert, M., Lee, H., Assländer, J., Zaitsev, M. and Hennig, J. , "Single shot concentric shells trajectories for ultra fast fMRI," Magnetic resonance in medicine 68(2), 484-494 (2012).

[16] Assländer, J., Zahneisen, B., Hugger, T., Reisert, M., Lee, H., LeVan, P. and Hennig, J. , "Single shot whole brain imaging using spherical stack of spirals trajectories," Neuroimage 73, 59-70 (2013).

[17] Jenkinson, M., Bannister, P., Brady, M. and Smith, S. , "Improved optimization for the robust and accurate linear registration and motion correction of brain images," Neuroimage 17(2), 825-841 (2002).

[18] Beckmann, C. F. and Smith, S. M. , "Probabilistic independent component analysis for functional magnetic resonance imaging," IEEE Trans.Med.Imaging 23(2), 137-152 (2004). 